\title{
Noninvasive metabolic profiling for painless diagnosis of human diseases and disorders
}

\begin{abstract}
Metabolic profiling provides a powerful diagnostic tool complementary to genomics and proteomics. The pain, discomfort and probable iatrogenic injury associated with invasive or minimally invasive diagnostic methods, render them unsuitable in terms of patient compliance and participation. Metabolic profiling of biomatrices like urine, breath, saliva, sweat and feces, which can be collected in a painless manner, could be used for noninvasive diagnosis. This review article covers the noninvasive metabolic profiling studies that have exhibited diagnostic potential for diseases and disorders. Their potential applications are evident in different forms of cancer, metabolic disorders, infectious diseases, neurodegenerative disorders, rheumatic diseases and pulmonary diseases. Large scale clinical validation of such diagnostic methods is necessary in future.
\end{abstract}

Lay abstract: Metabolic profiling involves determination of changes in metabolite levels caused by diseases and can help in their diagnosis. The pain, discomfort and chance of injury associated with painful diagnostic methods, make them unsuitable in terms of patient compliance. Metabolic profiling of body fluids like urine, breath, saliva, sweat and feces, which can be collected in a painless manner, could be used for painless diagnosis. This review article is about such studies, which have exhibited painless diagnostic potential for diseases and disorders. Their applications are evident in cancer, metabolic disorders, infections, neurodegenerative disorders, rheumatic diseases and lung diseases.

First draft submitted: 9 December 2015; Accepted for publication: 29 January 2016; Published online: 10 June 2016

Keywords: biomarkers $\bullet$ exhaled breath $\bullet$ feces $\bullet$ metabolic profiling $\bullet$ metabolomics

- noninvasive diagnosis $\bullet$ saliva $\bullet$ urine

\section{Background}

The idea of using qualitative metabolite patterns for noninvasive diagnosis of diseases using basic chromatographic techniques dates back to the late 1940s whereby characteristic metabolic patterns in urine and saliva were used for diagnosis [1]. However, the advancement of analytical platforms in the late 1960s ushered the advent of quantitative metabolic profiling. Since its inception the field of metabolic profiling has grown considerably in terms of its applications and contributions to systems biology research. Metabolic profiling is often described by closely related terms such as metabonomics, metabolic phenotyping, metabolomic fingerprinting and metabolomics which are often used interchangeably in contemporary scientific studies [2,3]. Metabolic profiling provides a powerful tool for gaining valuable insight into functional biology, toxicology, pharmacology and hence aids in diagnosis of diseases $[4,5]$. Metabolic profiling is complementary to genomics and proteomics as it

\section{Mainak Mal}

Freelance science writer, 40 T.C. Road, Flat \#2A, Kolkata 700053, India Tel.: +919038444883

mainakmal@gmail.com 
measures the perturbed metabolic end points due to genetic, environmental, pharmacological or pathological influences while in genomics and proteomics, more upstream biological events are typically profiled and studied [6]. It involves the analysis of various biological matrices using suitable analytical platforms. Metabolic profiling can be carried out with a global nontargeted approach as well as with a targeted approach. In targeted metabolic profiling, alterations in the levels of a specific class of metabolites or metabolites belonging to a specific metabolic pathway are ascertained using an appropriate analytical technique [7,8]. In global nontargeted metabolic profiling, metabolites belonging to diverse metabolic pathways are profiled. The metabolites that are determined in nontargeted approach belong to various chemical classes such as organic acids, amino acids, fatty acids, amines, sugars, sugar alcohols, steroids, nucleic acid bases, phospholipids and other miscellaneous substances. So, multiple complementary analytical techniques are often utilized for nontargeted metabolic profiling of biological matrices, in order to cover as much of metabolic space as possible. So, multiple complementary analytical techniques are often utilized for nontargeted metabolic profiling of biological matrices, in order to cover as much of metabolic space as possible [9,10]. Analytical platforms that are commonly used for metabolic profiling include NMR spectroscopy and MS based techniques like direct infusion MS, GC-MS, LC-MS or CE-MS. In addition to these techniques other methods like Fourier transform infrared (FTIR) spectroscopy, LC with ultraviolet or coulometric detection and CE with ultraviolet detection have also been used for metabolic profiling.

Once the biological samples for metabolic profiling are collected they are usually stored at $-80^{\circ} \mathrm{C}$ until further analysis to prevent sample degradation. Selection of sample preparation technique depends upon the type of analytical platform used for metabolic profiling. Although for NMR spectroscopy minimal sample pretreatment is required, the data generated are difficult to process and interpret. In case of LC-MSbased methods, sample cleanup step is required before analysis to eliminate matrix effect. GC-MS methods are not susceptible to matrix effect but lacks throughput because of the extensive sample preparation involved [7].

Global nontargeted metabolic profiling results in the generation of huge and complex datasets containing a large number of variables. Multivariate statistical techniques or chemometric tools are indispensable for the analysis of such datasets. Of the different chemometric methods available such as hierarchical clustering, partitional clustering, artificial neural networks, sup- port vector machine, evolutionary-based algorithms and regression trees, projection-based chemometric methods are extensively used [11].

In spite of the potential applications of metabolic profiling, it has some intrinsic limitations and shortfalls which limit its widespread adaptability in clinical practices. The main lacuna lies in the standardization of collection procedure of biological samples such as urine and feces as various factors like sex, age, life style factors, health status and physical exercise present challenges in analysis and interpretation of acquired data. Moreover selection of proper sample preparation techniques, analytical method development, raw data analysis and integration with other 'omic' sciences present various challenges in metabolic profiling [12-16].

Diagnostic methods using invasive and minimally invasive techniques have certain limitations and shortfalls of their own. For instance, endoscopic diagnostic techniques are invasive and may cause iatrogenic injury to patients [17]. Moreover, minimally invasive techniques such as collection of blood or tears, involve trained personnel and the pain associated renders it unpopular among the young population and even among certain adults. So there is an ardent necessity of developing noninvasive diagnostic techniques in order to improve patient compliance and thereby to encourage the active participation of patients for diagnosis.

Blood, urine and tissue are the commonly used biomatrices for metabolic profiling. Biomatrices such as urine, exhaled breath, saliva, sweat and fecal extract can be obtained in a noninvasive manner. Therefore metabolic profiling of these biomatrices holds immense potential and promise for noninvasive diagnosis of diseases [10]. Previously Medina et al. [18] authored a review which was focused mainly on the diagnostic metabolomic biomarkers and associated pathways identified only from clinical trials. This review article is an effort to collate and summarize both preclinical and clinical noninvasive metabolic profiling studies carried out so far to develop diagnostic methods of human diseases.

\section{Urine as a biomatrix for noninvasive metabolic profiling}

Urine sample collection is noninvasive by nature and is not restricted by volume. Urine can also be used for measurement of time-resolved, dynamic or temporal data which are an essential attribute for the investigation of the pathogenesis, progression and prognostication of acute and chronic diseases. The metabolite composition of urine is also influenced by other factors such as diet and physiological parameters such as age, gender and demographic characteristics. In some instances, urine may consist of xenobiotics and their metabolites, which can lead to additional complexities 
in downstream data analysis. Therefore, proper study design in urinary metabolic profiling is of utmost importance to acquire meaningful data and consequent interpretation. Baseline characteristics involved in study groups, sample collection and storage conditions should be carefully controlled and standardized according to the research hypothesis. Diurnal variations in urine caused by diet and lifestyle factors can be nullified by collection of first-pass urine [19].

Urine-based noninvasive metabolic profiling studies have been predominantly used for the diagnosis of different types of cancers such as prostate cancer, lung cancer, liver cancer, bladder cancer, gastric cancer and breast cancer (please refer to Table 1). Urine-based metabolomics in conjunction with other 'omics' was used for noninvasive diagnosis of prostate cancer and the method developed outperformed prostate-specific antigen in diagnosis potential [20]. Several studies have indicated that a panel of the urinary nucleosides identified with metabolic profiling performs better with cancer diagnosis than the traditional single tumor biomarker as evident in case of liver cancer [21], breast cancer [22,23] and lung cancer [24,25]. A noninvasive sensitive method to identify volatile organic metabolites as biomarkers that can accurately diagnose the onset of breast cancer was developed by Silva et al. [26]. Diagnostic methods of bladder cancer, like cystoscopy and urinary cytology, have a lot of shortfalls. As cystoscopy is expensive and invasive, it lacks patients' compliance and often fails in detecting recurrent bladder cancer. Urine-based noninvasive metabolic profiling has shown immense potential in replacing the existing conventional diagnostic methods even in distinguishing muscle invasive and nonmuscle invasive bladder cancer [27-30]. Large scale screening and diagnosis for gastric cancer using endoscopy lacks practicality because of the expenses involved, patient compliance and ease of administration. A urinary metabolic profiling approach was found to be useful for the effective diagnosis of gastric cancer and exhibited higher accuracy and sensitivity than carbohydrate antigen 19-9 and carcinoembryonic antigen. More- over, 4-hydroxyphenylacetate, alanine, phenylacetylglycine, mannitol, glycolate and arginine levels were significantly correlated with cancer $T$ stage and along with hypoxanthine level were able to discriminate healthy from the gastric cancer patients [31,32].

Urine-based noninvasive metabolic profiling studies have also exhibited immense potential in the diagnosis of human metabolic disorders as well as in born errors of metabolism in infants (please refer to Table 2) [33]. Metabolic profiling of infant urine was applied for the diagnosis of organic acidurias and relevant biomarker discovery [34]. Urinary metabolic profiling was also used for screening of 5-day-old newborns for neonatal galactosuria [35]. Nontargeted metabolic profiling by analysis of urine samples collected from infants has been successfully used in the diagnosis of some inborn metabolic disorders such as cystinuria, maple syrup urine disease, adenylosuccinate lyase deficiency and galactosemia [36]. In order to identify urinary biomarkers associated with nutritional rickets in children and to establish a noninvasive diagnosis method, urinary metabolic profiling along with metabolic pathway analysis was used to investigate the metabolic alterations associated with the disease [37]. Wei et al. carried out urinary metabolomic profiling for the identification of metabolites relevant for noninvasive diagnosis and stratification of prediabetic stage, which might be imperative in customized treatment for diabetics [38]. Although determination of phenylalanine/tyrosine ratio in blood has been conventionally utilized in diagnosis of phenylketonuria, there are limitations presented by its invasive nature and associated false positives. Recently, a urinary metabolic profiling-based diagnostic method for phenylketonuria has been developed to overcome these limitations [39].

Urine-based metabolic profiling has also been extensively used for noninvasive diagnosis of other diseases and conditions (please refer to Table 3). Gestational hypertensive disorders of pregnancy, especially preeclampsia might contribute to maternal morbidity. A combination of maternal characteristics with the noninvasive urinary metabolic profiling of

Table 1. Urinary metabolic profiling studies used for noninvasive diagnosis of different types of cancer.

\begin{tabular}{|llr|}
\hline Type of cancer & Analytical platform used & Ref. \\
Bladder cancer & LC-MS, GC-MS & {$[26-28]$} \\
\hline Breast cancer & HPLC, LC-MS, GC-MS & {$[22,23]$} \\
\hline Gastric cancer & GC-MS, NMR & {$[31,32]$} \\
Liver cancer & NMR, MS, LC-MS, GC-MS & {$[21]$} \\
\hline Lung cancer & NMR, MS, LC-MS, GC-MS & {$[24,25]$} \\
\hline Prostate cancer & LC-MS & {$[20]$} \\
\hline
\end{tabular}


Table 2. Urinary metabolic profiling studies used for noninvasive diagnosis of metabolic disorders and in born errors of metabolism.

\begin{tabular}{|c|c|c|}
\hline Diseases or disorders & Analytical platform used & Ref. \\
\hline $\begin{array}{l}\text { Cystinuria, maple syrup urine disease, adenylosuccinate lyase } \\
\text { deficiency, galactosemia }\end{array}$ & LC-MS & [3] \\
\hline Neonatal galactosuria & GC-MS & [35] \\
\hline Nutritional rickets & LC-MS & [37] \\
\hline Organic acidurias & GC-MS & [34] \\
\hline Phenylketonuria & GC-MS & [39] \\
\hline Prediabetic subtypes & GC-MS & [38] \\
\hline
\end{tabular}

hippurate/creatinine level significantly improved the prediction rates of preeclampsia $[40,41]$. A preliminary study by Blydt-Hansen $e t$ al. indicated that urinary metabolic profiling has the potential to be a sensitive, specific and noninvasive diagnostic method for T-cell-mediated rejection in pediatric kidney transplant recipients. This method was found to be better than the method involving serum creatinine, with minimal confounding parameters introduced by other allograft injury processes [42]. Crohn's disease along with ulcerative colitis makes up the majority of inflammatory bowel diseases (IBD). In the last few years, urinary metabolic profiling studies have been extensively utilized for gaining more insight into metabolic pathways perturbed in IBD which in turn has provided a potential diagnostic and patient stratification tool for IBD [43]. A preliminary study involving urinary metabolic profiling exhibited potential in the diagnosis of hepatitis $\mathrm{C}$ virus infection in patients [44]. Similarly a urinary metabolic profiling study exhibited potential for the clinical diagnosis of concomitant depression in hepatitis B virus infected patients. The six relevant biomarkers namely pyruvate, isobutyrate, $N$-methylnicotinamide, alpha-hydroxybutyrate, acetoacetate and malonate might be helpful for developing a validated diagnostic method for the above purpose [45]. Dipstick urinalysis is the conventional semi-quantitative method for diagnosis urinary tract infection but lacks diagnostic accuracy [46]. A recent study by Lam et al. involving urine-based targeted metabolic profiling of microbial-mammalian co-metabolite trimethylamine demonstrated its potential for the etiological diagnosis of Escherichia coli associated urinary tract infection [47]. Pike et al. demonstrated the auxillary role of urinary steroid metabolic profiling in the diagnosis of familial precocious puberty, a subgroup of true precocious puberty [48]. Clinical and preclinical studies involving murine models have already demonstrated the potential of urinary metabolic profiling in improving the diagnosis of rheumatic diseases such as rheumatoid arthritis, spondyloarthritis, systemic lupus erythematosus and osteoarthritis [49]. Urinary metabolic profiling has been successfully utilized in the diagnosis of interstitial cystitis and bacterial cystitis [50].

Table 3. Urinary metabolic profiling studies used for noninvasive diagnosis of miscellaneous diseases and disorders.

\begin{tabular}{llr} 
Diseases or disorders & Analytical platform used & Ref. \\
\hline $\begin{array}{llr}\text { Depression associated with hepatitis B virus infection } \\
\text { Familial precocious puberty }\end{array}$ & NMR & {$[45]$} \\
\hline $\begin{array}{l}\text { Gestational hypertensive disorder especially } \\
\text { preeclampsia }\end{array}$ & NMR & {$[48]$} \\
\hline Hepatitis C virus infection & NMR & {$[41]$} \\
\hline Immuno-rejection in kidney transplantation & MS & {$[44]$} \\
Inflammatory bowel diseases & NMR, MS, LC-MS, GC-MS & {$[42]$} \\
\hline Interstitial and bacterial cystitis & NMR, MS & {$[43]$} \\
\hline $\begin{array}{l}\text { Rheumatoid arthritis, spondyloarthritis, systemic } \\
\text { lupus erythematosus, osteoarthritis }\end{array}$ & NMR, MS & {$[50]$} \\
Urinary tract infection & NMR & {$[49]$} \\
\hline
\end{tabular}




\section{Saliva as a biomatrix for noninvasive metabolic profiling}

The ease, cost-effectiveness and noninvasive nature of collection, make saliva an ideal bio-matrix for noninvasive metabolic profiling studies. Its functional biology in the oral cavity is well established. Advances in metabolic profiling have resulted in the identification and characterization of an array of salivary metabolites such as amino acids, lipids, antioxidants, polyamines, vitamins and ethylphosphate [51]. These metabolites enter the saliva through extracellular and intracellular routes, carrying real-time information from several organs and thereby provide a potential means of diagnosis of diseases. Saliva is relatively free from confounding factors generally involved in metabolic profiling, such as hemoglobin level, blood pressure, gender and BMI thus making it an ideal bio-matrix for attaining accurate realtime diagnostic results. However, the salivary metabolic profile is influenced by factors such as smoking [52] and diurnal variation [53]. For the development of basic, translational and clinical research in saliva, bioinformatics tools such as 'Saliva Ontology', 'SDxMart' as well as databases such as 'Saliva-omics Knowledge Base' developed by the National Institute of Dental and Craniofacial Research, USA and the National Cancer Institute, USA are available. This has provided efficient data management system and web resources, which in turn has boosted research in salivary metabolic profiling [54]. These factors have contributed to the expansion of the range of salivary metabolic profiling for the diagnosis of both oral and systemic diseases. Salivary metabolic profiling is applicable for diagnosis of local diseases such as oral cancers, Sjogren's syndrome, periodontal diseases as well as distant diseases like autoimmune, metabolic diseases, viral/bacterial infections (please refer to Table 4). Moreover, this provides a means for the development of effective diagnostic devices for clinical application [55-59].
Salivary metabolic profiling has resulted in the identification of unique metabolic signatures of carbohydrate, lipid metabolism and oxidative stress associated with periodontal diseases such as gingivitis and periodontitis, which are associated with Type 2 diabetes mellitus. This in turn could be used for diagnosis and monitoring of both diabetes and periodontal disease [61]. Moreover, other salivary metabolic profiling studies indicated a strong association of Type 2 diabetes with individual salivary biomarkers such as 1,5-anhydroglucitol [63] and D/L-Lactic acid isomer ratio [64]. This could be used as noninvasive diagnostic biomarkers for Type 2 diabetes mellitus.

Salivary metabolic profiling has led to the identification of potential diagnostic biomarkers for Sjogren's syndrome. Sjogren's syndrome is characterized by chronic inflammation and destruction of salivary glands. The study captured the associated perturbations in salivary metabolite pattern, especially a reduction in the level of marker metabolites such as glycine, tyrosine, uric acid, fucose etc. as compared with health controls [65].

Salivary metabolic profiling in conjunction with serum based metabolic profiling was able to provide diagnostic biomarkers of neurodegenerative dementia such as Alzheimer's disease, frontotemporal lobe dementia and Lewy body disease [66].

Early diagnosis of oral squamous cell carcinoma and associated precancerous lesions such as oral lichen planus and oral leukoplakia is essential for increasing the survival rate of patients. Several studies utilizing salivary metabolic profiling have demonstrated the diagnostic potential of several metabolite based biomarkers like propionylcholine, $N$-Acetyl-L-phenylalanine, sphinganine, phytosphingosine, S-carboxymethyl-L-cysteine, $\gamma$-aminobutyrate, phenylalanine, valine, $n$-eicosanoic acid and lactate [67-69]. Apart from oral cancer metabolic profiling of saliva samples has

Table 4. Salivary metabolic profiling studies used for noninvasive diagnosis of different diseases and disorders.

\begin{tabular}{|c|c|c|}
\hline Diseases or disorders & Analytical platform used & Ref. \\
\hline Breast cancer & CE-MS & [60] \\
\hline $\begin{array}{l}\text { Neurodegenerative dementias (Alzheimer's disease, } \\
\text { frontotemporal lobe dementia, Lewy body disease) }\end{array}$ & CE-MS & [57] \\
\hline Pancreatic cancer & CE-MS & [60] \\
\hline $\begin{array}{l}\text { Periodontal diseases like (gingivitis and periodontitis) in } \\
\text { diabetic patients }\end{array}$ & GC-MS, LC-MS & [53] \\
\hline Primary Sjogren's syndrome & GC-MS, LC-MS & {$[55,56]$} \\
\hline $\begin{array}{l}\text { Squamous cell carcinoma and related precancerous lesions } \\
\text { like oral lichen planus and oral leukoplakia }\end{array}$ & LC-MS, CE-MS & {$[58-59,61,62]$} \\
\hline Type 2 diabetes mellitus & NMR, MS, LC-MS, GC-MS & [54] \\
\hline
\end{tabular}


also resulted in the identification of disease-specific markers of pancreatic cancer and breast cancer [60].

\section{Exhaled breath as a biomatrix for noninvasive metabolic profiling}

The fast, accurate and noninvasive diagnosis of respiratory diseases comprises a challenge to the clinicians, and the clinical management can be complicated by the lack of knowledge of the different types of respiratory diseases. Exhaled breath contains a complex mixture of volatile organic compounds (VOCs) in the gas phase which are generated by respiratory tract itself or pulmonary circulation. Thus they could potentially represent metabolite-based diagnostic biomarkers for lung diseases. For instance metabolic profiling of noninvasive VOCs has been used to diagnose lung cancer, infectious diseases, asthma and chronic obstructive lung diseases (please refer to Table 5). Exhaled breath condensate metabolite analysis could be also used as a noninvasive method for the study of pleural fluid. One of the confounding factors associated with the analysis of exhaled breath is the high amount of moisture present in the biomatrix. Moreover the diagnostic accuracy of exhaled breath analysis for some lung diseases like acute respiratory distress syndrome (ARDS) has been validated by preclinical murine models [70]. However, the main limitations to its applicability in regular clinical practice are the standardization of baseline characteristics in study populations as well as establishment of standard collection and analytical procedures [71-79]. Collection of exhaled breath usually utilizes electronic nose such as gold nanoparticles and sensors-based electronic nose. Breath samples are usually stored in different containers with Tedlar bag being the most common [77].

Basanta, et al. demonstrated that it was possible to distinguish patients with and without chronic obstructive pulmonary disease from healthy controls using noninvasive metabolic profiling of exhaled breath [72]. A need for diagnostic biomarkers of the ARDS necessitated metabolic profiling study of exhaled breath of patients. The study was able to identify three breath metabolites namely octane, acetaldehyde and 3-methylheptane which could be used for diagnosis of ARDS [76].

Metabolic profiling study of exhaled breath condensate in children was applied successfully to differentiate asthma phenotypes, with a particular focus on severe inflammatory state. Metabolites related to retinoic acid, adenosine and vitamin were found to be significant for separation of different asthma subtypes. In a similar study, a panel of eight metabolite based biomarkers, namely, 1-(methylsulfanyl)-propane, ethylbenzene, 1,4-dichlorobenzene, 4-isopropenyl-1-methylcyclohexene, 2-octenal, octadecyne, 1-isopropyl-3-methylbenzene and 1,7-dimethylnaphtalene were found to be relevant for effective separation of asthmatic and healthy children [80-84].

Early diagnosis of lung cancer is desirable for reduction of high rate of morbidity associated with the disease. Metabolic profiling of exhaled breath VOCs could provide a noninvasive method for widespread screening for early diagnosis of lung cancer. In vitro cell culture under hypoxic conditions, have been used for gaining knowledge and identification of biomarkers relevant to lung cancer diagnosis [90]. Clinical extrapolation of metabolic profiling studies of exhaled breath VOCs [79] have lead to the identification of diagnostic biomarkers of lung cancer such as n-Dodecane [91], 1-butanol, 3-hydroxy-2-butanone (for nonsmall cell lung cancer) [92], hexadecanal [93], 5-(2-methyl-)-propyl-nonane, 2,6-di-tert-butyl-4-methyl-phenol, 2,6,11-trimethyldodecane; 8-hexyl-hexadecanal; pentadecane [94]. Similar diagnostic applicability of metabolic profiling

Table 5. Metabolic profiling studies using exhaled breath for noninvasive diagnosis of different diseases and disorders.

\begin{tabular}{|llr|}
\hline Diseases or disorders & Analytical platform used & Ref. \\
\hline Acute respiratory distress syndrome & GC-MS & {$[68]$} \\
\hline Asthma & NMR & {$[80-84]$} \\
Breast cancer & GC-MS & {$[85,86]$} \\
\hline Chronic obstructive pulmonary disease & GC-DMS, NMR & {$[71,72]$} \\
\hline Cystic fibrosis and related diabetes & IMS-TOF-MS, NMR & {$[87,88]$} \\
\hline Hepatocellular carcinoma & GC-MS & {$[89]$} \\
\hline Lung cancer & GC-MS & {$[79,90-94]$} \\
\hline Pleural mesothelioma & GC-MS & {$[95]$} \\
\hline Primary ciliary dyskinesia & NMR & {$[96]$} \\
\hline Pulmonary Langerhans cell histiocytosis & NMR & {$[62]$} \\
\hline GC-DMS: Gas-chromatography-differential mobility spectrometry; IMS-TOF-MS: lon-mobility-time of flight-mass spectrometry.
\end{tabular}


Table 6. Metabolic profiling studies using feces for noninvasive diagnosis of different diseases and disorders.

\begin{tabular}{|llr}
\hline Diseases or disorders & Analytical platform used & Ref. \\
\hline Irritable bowel syndrome and Inflammatory bowel diseases & GC-MS, NMR & {$[101,102]$} \\
\hline Colorectal cancer & GC-MS, NMR & {$[103-105]$} \\
\hline Type-2 diabetes mellitus & FT-ICR-MS, LC-MS & {$[106]$} \\
\hline FT-ICR-MS: Fourier transform-Ion cyclotron resonance-Mass spectrometer. & & \\
\hline
\end{tabular}

of exhaled breath can be found in case of pleural mesothelioma [95].

Metabolic profiling of exhaled breath has shown noninvasive diagnostic potential in other forms of cancer such as breast cancer and liver cancer. The diagnostic potential of exhaled breath metabolites in breast cancer have been demonstrated by some studies. These have resulted in the identification of significant diagnostic biomarkers such as 3-methylhexane, dec-1-ene, caryophyllene, naphthalene, trichloroethene [85], hexanal, heptanal, octanal and nonanal [86]. Metabolic profiling of exhaled breath has also been used for noninvasive diagnosis of liver cancer and has resulted in the identification of diagnostic biomarkers such as 3-Hydroxy2-butanone, hepatocellular carcinoma and decane [89].

Metabolic profiling of breath VOCs has been utilized to separate breath metabolite patterns of patients with and without lower respiratory tract infections thus exhibiting its diagnostic potential in respiratory tract infections [97].

Metabolic profiling of exhaled breath condensate was used to discriminate cystic fibrosis (CF) from healthy controls thus exhibiting its diagnostic potential [87]. CFrelated diabetes (CFRD) may result from $\mathrm{CF}$ and it is characterized by rapid decline in lung condition. Moreover, targeted metabolic profiling of glucose in exhaled breath condensate has the potential of successfully diagnosing CF and CFRD [88]. NMR-based metabolic profiling studies have been utilized for differentiating primary ciliary dyskinesia from CF [96] as well as patients suffering from pulmonary Langerhans cell histiocytosis from healthy subjects [62].

\section{Feces as a biomatrix for noninvasive metabolic profiling}

Fecal samples consist of diverse range of endogenous metabolites, gut microbiota metabolites with different physicochemical properties and volatility. Metabolic profiling of feces not only helps in the discovery of disease biomarkers but also in providing an insight about the relationship of the gut microbiome and human health. The transient stay of feces in the colon and rectum makes it an ideal biomatrix for gaining information about the health and pathological state of colon and rectum. Metabolic profiling of feces has the poten- tial for noninvasive diagnosis of IBD and colorectal cancer (please refer to Table 6). However, the complex nature of feces present challenges in sample collection, sample storage, sample preparation and development of analytical methods [98-100].

Metabolic profiling of fecal volatile organic metabolites helped in providing information about the etiology of the disease and also resulted in the identification of diagnostic biomarkers of irritable bowel syndrome [101]. Crohn's disease along with ulcerative colitis makes up the majority of IBD. Discrimination of Crohn's disease from ulcerative colitis as well differentiation of IBD from irritable bowel syndrome is imperative for patient stratification and effective clinical management. However, conventional diagnostic methods lack accuracy in this matter. Metabolic profiling studies of feces have already shown diagnostic potential in case of the above-mentioned bowel conditions [101,102].

Colorectal cancer is one of the major forms of cancer in terms of morbidity in developed countries. The application of metabolic profiling to fecal water extracts has shown potential as a diagnostic tool for detecting colorectal cancer. NMR-based metabolic profiling of fecal water extracts from patients with colorectal cancer and healthy individuals, was able to identify potential diagnostic markers such as short-chain fatty acids (acetate and butyrate) and amino acids (proline and cysteine) [103]. A similar study using lyophilized feces resulted in the identification of relevant fecal biomarkers of colorectal cancer [104]. Metabolic profiling of fecal samples using GC-MS also exhibited diagnostic potential in the detection of colorectal cancer [105].

$\mathrm{The} \mathrm{db} / \mathrm{db}$ mouse model, consisting of a nonfunctional leptin receptor, is a commonly used murine model of Type 2 diabetes mellitus. Nontargeted metabolic profiling of fecal extracts of $\mathrm{db} / \mathrm{db}$ and wild-type mice revealed a wide range of metabolites related to diabetes such as sulfate containing fatty acids (cyprinol sulfate), sulfate containing bile acids (sulfocholic acid, oxocholic acid sulfate, taurocholic acid sulfate) and steroidal metabolites. These metabolites were able to discriminative wild-type from $\mathrm{db} / \mathrm{db}$ mice. Furthermore, including $\mathrm{N}$-acyl taurines were altered in diabetic mice, enabling us to focus on S-containing metabolites, especially the sulfate and taurine conjugates of bile and fatty acids [106]. 
Unconventional biomatrices for noninvasive metabolic profiling

Metabolic profiling of hair samples collected from three different rodent model namely hypertensive model rats (SHR/Izm), stroke-prone SHR
(SHRSP/Izm) and Wistar Kyoto (WKY/Izm) was carried out using LC-MS. The results obtained were subjected to chemometric analysis which in turn resulted in successful group separation. This study demonstrated the future potential of hair as a feasible

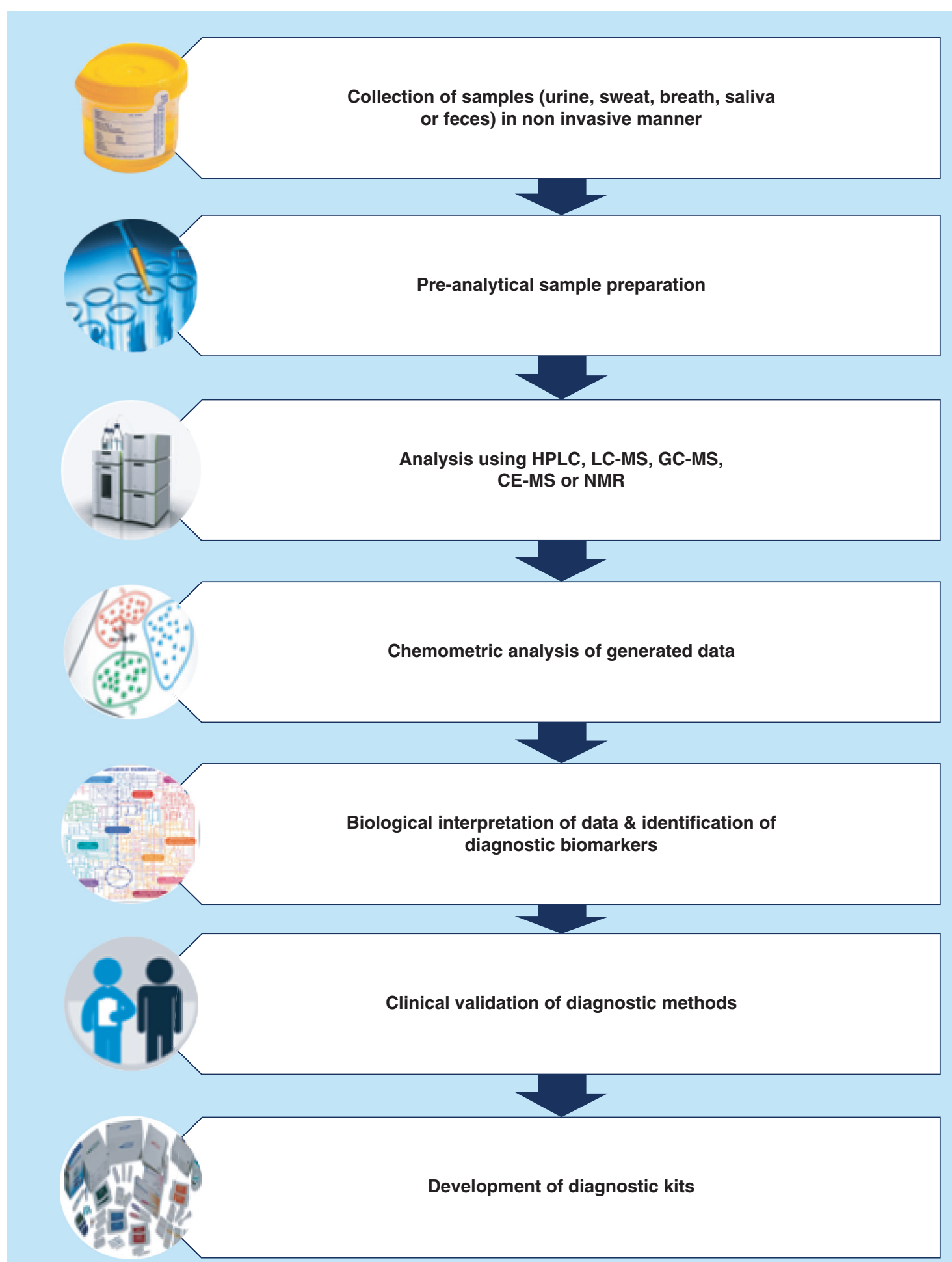

Figure 1. General workflow for development of painless diagnostic methods using metabolic profiling. 
biomatrix for noninvasive metabolic profiling for diagnostic purpose [107].

Sweat is an unconventional biomatrix when it comes to biomarker discovery and diagnosis of diseases. So far sweat electrolyte concentration has been successfully used for the diagnosis of CF [108]. Recently, metabolic profiling of sweat has been carried out for noninvasive diagnosis of lung cancer. This resulted in the identification of significant marker metabolites such as a trisaccharide phosphate, suberic acid, a tetrahexose, a trihexose, nonanedioic acid and monoglyceride (22:2) [109].

\section{Conclusion}

Noninvasive diagnostic methods are essential for clinical management of human diseases and disorders from the point of view of patient compliance and comfort. A general workflow for the development of painless diagnostic methods using metabolic profiling has been shown in Figure 1. The information provided in this review affirmed the established as well as the potential applications of metabolic profiling in the noninvasive diagnosis of human diseases and disorders. The most commonly used biomatrices that are collected and used for this purpose are urine, exhaled breath, saliva and fecal extract [10]. Apart from these popular biomatrices, there exist some unconventional biomatrices such as sweat [109] and hair [107] that can be used for noninvasive diagnostics. The application of metabolic profiling for noninvasive diagnostics can be observed in different forms of cancer, metabolic disorders like diabetes, in born errors of metabolism, infectious diseases, neurodegenerative disorders rheumatic diseases and pulmonary diseases. Preclinical studies and small scale clinical studies have provided evidence of the diagnostic potential of such metabolic profiling studies. However, further large scale clinical studies are essential for validation of some diagnostic methods and to extend its application in regular clinical practice. Therefore, metabolic profiling of biomatrices which can be collected noninvasively holds the key for painless diagnosis of diseases and disorders.

\section{Future perspective}

The diagnostic potential of metabolic profiling of biomatrices which can be collected painlessly in certain diseases and disorders has been asserted strongly by several preclinical and small scale clinical studies. The predictive power of the noninvasive diagnostic methods depends upon study design, baseline characteristics and confounding factors which make such comparison and meta-analysis difficult to establish. So, clinical studies are required in future for validation of the potential diagnostic methods. For the implementation of metabolic profiling as a nonnvasive diagnostic tool in clinical practices large scale prospective clinical stud- ies are required in distant future. The main thrust areas involve diseases such as IBD [43], hepatitis $C$ virus infection [44], depression associated with hepatitis $\mathrm{B}$ virusinfected patients [45], neurodegenerative disorders [66], ARDS [76], pleural mesothelioma [95], respiratory tract infections [97], nonalcoholic fatty liver disease [110,111] and rheumatic diseases [49].

As metabotype varies from one biological sample to another as well as between different studies, validation of multivariate statistical model for a particular disease or disorder is of utmost importance. Validation of the already available results especially in the field of metabolic profiling of saliva, exhaled breath and urine could lead to the development of high-throughput noninvasive point-of-care and bedside diagnostic devices. Development of such devices are feasible in some forms of cancer like breast cancer, lung cancer, oral cancer and pancreatic cancer [77,105] and gestational hypertensive disorders especially preeclampsia $[40,41]$.

Future research in the field of metabolic profiling using unconventional biomatrices such as sweat and hair could increase the popularity of these biomatrices in terms of their diagnostic applications [107,108]. The tear film covering the epithelial cells of the ocular surface is essential for visual function and its composition is a good indicator of the surface-health of the eye. However, the clinical method of tear collection is minimally invasive and results in confounding factors in metabolic profiling. All these have restricted its feasibility as a biomatrix for noninvasive metabolic profiling. However, innovation in the collection method of tear samples could minimize the associated discomfort and could extend its utility for noninvasive diagnosis of eye diseases $[112,113]$. In the future, research in sensor technologies could also help in the advancement of point-ofcare diagnostic devices especially in the field of exhaled breath metabolic profiling. Thus noninvasive metabolic profiling has paved the way for an exciting field of future research in diagnostic methods and devices.

\section{Financial \& competing interests disclosure}

The author has no relevant affiliations or financial involvement with any organization or entity with a financial interest in or financial conflict with the subject matter or materials discussed in the manuscript. This includes employment, consultancies, honoraria, stock ownership or options, expert testimony, grants or patents received or pending, or royalties.

No writing assistance was utilized in the production of this manuscript.

\section{Open access}

This work is licensed under the Creative Commons Attribution 4.0 License. To view a copy of this license, visit http://creativecommons.org/licenses/by/4.0/ 


\section{Background}

- Painful diagnostic methods using invasive or minimally invasive techniques may cause patient discomfort and iatrogenic injury to patients.

- There is a need for development of noninvasive diagnostic methods in order to improve patient compliance and patient participation.

- Metabolic profiling of biomatrices like urine, exhaled breath, saliva, sweat and feces which can be collected in a painless manner could be used for noninvasive diagnosis of diseases and disorders.

Urine for noninvasive metabolic profiling

- Urinary metabolic profiling studies have been predominantly used for the diagnosis of different forms of cancers, metabolic disorders and in born errors of metabolism.

- Urinary metabolic profiling has also been used for noninvasive diagnosis of gestational preeclampsia, infectious diseases and rheumatic diseases.

Saliva for noninvasive metabolic profiling

- Salivary metabolic profiling is applicable for diagnosis of local diseases like oral cancers, Sjogren's syndrome and periodontal diseases.

- The applicability of salivary metabolic profiling could also be extended for the diagnosis autoimmune disorders, metabolic diseases and infectious diseases.

Exhaled breath for noninvasive metabolic profiling

- Exhaled breath consists of a complex mixture of volatile organic compounds in the gas phase and could act as diagnostic biomarkers for diseases.

- Metabolic profiling of exhaled breath has been used to diagnose lung cancer, liver cancer, chronic obstructive lung diseases, cystic fibrosis and respiratory tract infections.

Feces for noninvasive metabolic profiling

- Feces provide information about the interaction of the gut microbiome and human health.

- The transient stay of feces in colon makes it an ideal biomatrix for gaining information about its health and pathological state.

- Metabolic profiling of feces has the potential for the diagnosis of irritable bowel syndrome, inflammatory bowel diseases and colorectal cancer.

Unconventional biomatrices for noninvasive metabolic profiling

- Metabolic profiling of unconventional biomatrices such as hair and sweat holds future potential for noninvasive diagnosis of diseases and disorders.

Conclusion

- The most commonly used biomatrices include urine, exhaled breath, saliva and feces in addition to less popular biomatrices like sweat and hair.

- The application of metabolic profiling for noninvasive diagnostics can be observed in cancer, metabolic disorders, in born errors of metabolism, infectious diseases, neurodegenerative disorders, rheumatic diseases and pulmonary diseases.

- Future large scale clinical studies are necessary for validation of some diagnostic methods and to extend its application in regular clinical practice.

\section{References}

Papers of special note have been highlighted as:

- of interest; $\bullet$ of considerable interest

1 Gates SC, Sweeley CC. Quantitative metabolic profiling based on gas chromatography. Clin. Chem. 24(10), 1663-1673 (1978).

2 Holmes E, Wilson ID, Nicholson JK. Metabolic phenotyping in health and disease. Cell 134(5), 714-717 (2008).

3 Kosmides AK, Kamisoglu K, Calvano SE, Corbett SA, Androulakis IP. Metabolomic fingerprinting: challenges and opportunities. Crit. Rev. Biomed. Eng. 41(3), 205-221 (2013).

4 Nicholson JK, Lindon JC, Holmes E. 'Metabonomics': understanding the metabolic responses of living systems to pathophysiological stimuli via multivariate statistical analysis of biological NMR spectroscopic data. Xenobiotica 29(11), 1181-1189 (1999).

5 Harrigan GG, Goodacre R. Metabolic profiling: its role in biomarker discovery and gene function analysis. Kluwer Academic Publishers, Boston, MA, USA (2003).

6 Fiehn O. Combining genomics, metabolome analysis, and biochemical modelling to understand metabolic networks. Comp. Funct. Genomics 2(3), 155-168 (2001).

7 Morris M, Watkins SM. Focused metabolomic profiling in the drug development process: advances from lipid profiling. Curr. Opin. Chem. Biol. 9(4), 407-412 (2005).

8 Urpi-Sarda M, Monagas M, Khan N et al. Targeted metabolic profiling of phenolics in urine and plasma after regular consumption of cocoa by liquid chromatographytandem mass spectrometry. J. Chromatogr. A 1216(43), 7258-7267 (2009). 
9 Dunn WB, Bailey NJ, Johnson HE. Measuring the metabolome: current analytical technologies. Analyst 130(5), 606-625 (2005).

10 Lindon JC, Holmes E, Nicholson JK. Metabonomics in pharmaceutical R\&D. FEBS J. 274(5), 1140-1151 (2007).

11 Goodacre R, Broadhurst D, Smilde AK et al. Proposed minimum reporting standards for data analysis in metabolomics. Metabolomics 3(3), 231-241 (2007).

12 Scalbert A, Brennan L, Fiehn O et al. Mass-spectrometrybased metabolomics: limitations and recommendations for future progress with particular focus on nutrition research. Metabolomics 5(4), 435-458 (2009).

13 Monteiro MS, Carvalho M, Bastos ML, Guedes De Pinho P. Metabolomics analysis for biomarker discovery: advances and challenges. Curr. Med. Chem. 20(2), 257-271 (2013).

14 Emwas AH, Luchinat C, Turano P et al. Standardizing the experimental conditions for using urine in NMR-based metabolomic studies with a particular focus on diagnostic studies: a review. Metabolomics 11(4), 872-894 (2015).

15 Emwas AH, Roy R, McKay RT et al. Recommendations and standardization of biomarker quantification using NMR-based metabolomics with particular focus on urinary analysis. J. Proteome Res. doi:10.1021/acs. jproteome.5b00885 (2016) (Epub ahead of print).

16 Klupczynska A, Derezinski P, Kokot ZJ. Metabolomics in medical sciences - trends, challenges and perspectives. Acta Pol. Pharm. 72(4), 629-641 (2015).

17 Brenner DE, Rennert G. Fecal DNA biomarkers for the detection of colorectal neoplasia: attractive, but is it feasible? J. Natl Cancer Inst. 97(15), 1107-1109 (2005).

18 Medina S, Dominguez-Perles R, Gil JI, Ferreres F, GilIzquierdo A. Metabolomics and the diagnosis of human diseases - a guide to the markers and pathophysiological pathways affected. Curr. Med. Chem. 21(7), 823-848 (2014).

- Provides information on the diagnostic metabolomic biomarkers and associated pathways identified from clinical trials.

19 Chan EC, Mal M, Pasikanti KK. Metabonomics. In: Handbook of Separation Science: Gas Chromatography. Poole CF (Ed). Elsevier, PA, USA, 545-562 (2012).

20 Cao DL, Ye DW, Zhang HL, Zhu Y, Wang YX, Yao XD. A multiplex model of combining gene-based, protein-based, and metabolite-based with positive and negative markers in urine for the early diagnosis of prostate cancer. Prostate 71(7), 700-710 (2011).

21 Yang J, Xu G, Zheng Y et al. Diagnosis of liver cancer using HPLC-based metabonomics avoiding false-positive result from hepatitis and hepatocirrhosis diseases. J. Chromatogr. B. Anal. Technol. Biomed. Life Sci. 813(1-2), 59-65 (2004).

22 Frickenschmidt A, Frohlich H, Bullinger D et al. Metabonomics in cancer diagnosis: mass spectrometry-based profiling of urinary nucleosides from breast cancer patients. Biomarkers 13(4), 435-449 (2008).

23 Fan X, Bai J, Shen P. Diagnosis of breast cancer using HPLC metabonomics fingerprints coupled with computational methods. Conf. Proc. IEEE Eng. Med. Biol. Soc. 6, 6081-6084 (2005).
24 Wang WZ, Zhao XJ, Li X, Chen J, Li FL, Xu GW. [Lung cancer diagnosis based on urinary modified nucleoside metabolic profiling]. Zhongguo Yi Xue Ke Xue Yuan Xue Bao. 29(6), 738-741 (2007).

25 Duarte IF, Rocha CM, Gil AM. Metabolic profiling of biofluids: potential in lung cancer screening and diagnosis. Expert Rev. Mol. Diag. 13(7), 737-748 (2013).

26 Silva CL, Passos M, Camara JS. Solid phase microextraction, mass spectrometry and metabolomic approaches for detection of potential urinary cancer biomarkers - a powerful strategy for breast cancer diagnosis. Talanta 89, 360-368 (2012).

27 Pasikanti KK, Esuvaranathan K, Ho PC et al. Noninvasive urinary metabonomic diagnosis of human bladder cancer. J. Proteome Res. 9(6), 2988-2995 (2010).

28 Jin X, Yun SJ, Jeong P, Kim IY, Kim WJ, Park S. Diagnosis of bladder cancer and prediction of survival by urinary metabolomics. Oncotarget 5(6), 1635-1645 (2014).

29 Hong Y, Esuvaranathan K, Pasikanti KK, Yong Chan EC. Metabonomic diagnosis of bladder cancer: what are the advantages and considerations? Bioanalysis 7(21), 2727-2729 (2015).

30 Shen C, Sun Z, Chen D et al. Developing urinary metabolomic signatures as early bladder cancer diagnostic markers. Omics 19(1), 1-11 (2015).

31 Jung J, Jung $Y$, Bang EJ et al. Noninvasive diagnosis and evaluation of curative surgery for gastric cancer by using NMR-based metabolomic profiling. Annals Surg. Oncol. 21(Suppl. 4), S736-S742 (2014).

32 Furina RR, Ryzhkov VL, Mitrakova NN et al. [The method for early diagnosis of the gastric cancer based metabolomics research]. Eksp. Klin. Gastroenterol. 10, 14-17 (2014).

33 Vaidyanathan K. Urinary proteomics and metabolomics in the diagnosis of pediatric disorders. Proteomics Clin. Appl. 9(5-6), 482-489 (2015).

34 Kouremenos KA, Pitt J, Marriott PJ. Metabolic profiling of infant urine using comprehensive two-dimensional gas chromatography: Application to the diagnosis of organic acidurias and biomarker discovery. J. Chromatogr. A 1217(1), 104-111 (2010).

35 Shinka T, Inoue Y, Peng H, Zhen-Wei X, Ose M, Kuhara T. Urine screening of five-day-old newborns: metabolic profiling of neonatal galactosuria. J. Chromatogr. B Biomed. Sci. Appl. 732(2), 469-477 (1999).

36 Janeckova H, Kalivodova A, Najdekr L et al. Untargeted metabolomic analysis of urine samples in the diagnosis of some inherited metabolic disorders. Biomedical Pap. Med. Fac. Univ. Palacky Olomouc Czech. Repub. 159(4), 582-585 (2014).

37 Wang M, Yang X, Ren L et al. Biomarkers identified by urinary metabonomics for noninvasive diagnosis of nutritional rickets. J. Proteome Res. 13(9), 4131-4142 (2014).

38 Wei H, Pasman W, Rubingh C et al. Urine metabolomics combined with the personalized diagnosis guided by Chinese medicine reveals subtypes of pre-diabetes. Mol. Biosyst. 8(5), 1482-1491 (2012).

39 Xiong X, Sheng X, Liu D, Zeng T, Peng Y, Wang Y. A GC/ MS-based metabolomic approach for reliable diagnosis of 
phenylketonuria. Anal. Bioanal. Chem. 407(29), 8825-8833 (2015).

40 Kenny LC, Broadhurst DI, Dunn W et al. Robust early pregnancy prediction of later preeclampsia using metabolomic biomarkers. Hypertension 56(4), 741-749 (2010).

41 Austdal M, Tangeras LH, Skrastad RB et al. First trimester urine and serum metabolomics for prediction of preeclampsia and gestational hypertension: a prospective screening study. Int. J. Mol. Sci. 16(9), 21520-21538 (2015).

42 Blydt-Hansen TD, Sharma A, Gibson IW, Mandal R, Wishart DS. Urinary metabolomics for noninvasive detection of borderline and acute $\mathrm{T}$ cell-mediated rejection in children after kidney transplantation. Am. J. Transplant. 14(10), 2339-2349 (2014).

43 De Preter V. Metabolomics in the clinical diagnosis of inflammatory bowel disease. Digest. Dis. 33(Suppl. 1), 2-10 (2015).

44 Godoy MM, Lopes EP, Silva RO et al. Hepatitis C virus infection diagnosis using metabonomics. J. Viral Hepatitis 17(12), 854-858 (2010).

45 Hou LJ, Wang HW, Wei XX et al. Urinary metabonomics for diagnosis of depression in hepatitis B virus-infected patients. Iran. Red Crescent Med. J. 17(4), e27359 (2015).

46 Lam CW, Law CY, To KK et al. NMR-based metabolomic urinalysis: a rapid screening test for urinary tract infection. Clin. Chim. Acta 436, 217-223 (2014).

47 Lam CW, Law CY, Sze KH, To KK. Quantitative metabolomics of urine for rapid etiological diagnosis of urinary tract infection: evaluation of a microbialmammalian co-metabolite as a diagnostic biomarker. Clin. Chim. Acta 438, 24-28 (2015).

48 Pike AW, Klein JL, Gotlin RW, Fennessey PV. The role of steroid metabolic profiling as an aid in the diagnosis of familial precocious puberty, a subgroup of true precocious puberty. J. Inherit. Metabol. Dis. 9(2), 147-155 (1986).

49 Priori R, Scrivo R, Brandt J et al. Metabolomics in rheumatic diseases: the potential of an emerging methodology for improved patient diagnosis, prognosis, and treatment efficacy. Autoimmun. Rev. 12(10), 1022-1030 (2013).

50 Van QN, Klose JR, Lucas DA et al. The use of urine proteomic and metabonomic patterns for the diagnosis of interstitial cystitis and bacterial cystitis. Dis. Markers 19(4-5), 169-183 (2003).

51 Alvarez-Sanchez B, Priego-Capote F, Luque De Castro MD. Study of sample preparation for metabolomic profiling of human saliva by liquid chromatography-time of flight/mass spectrometry. J. Chromatogr. A 1248 178-181 (2012).

52 Mueller DC, Piller M, Niessner R, Scherer M, Scherer G. Untargeted metabolomic profiling in saliva of smokers and nonsmokers by a validated GC-TOF-MS method. J. Proteome Res. 13(3), 1602-1613 (2014).

53 Bertram HC, Eggers N, Eller N. Potential of human saliva for nuclear magnetic resonance-based metabolomics and for health-related biomarker identification. Anal. Chem. 81(21), 9188-9193 (2009).
54 Ai JY, Smith B, Wong DT. Bioinformatics advances in saliva diagnostics. Int. J. Oral Sci. 4(2), 85-87 (2012).

55 Zhang A, Sun H, Wang X. Saliva metabolomics opens door to biomarker discovery, disease diagnosis, and treatment. Appl. Biochem. Biotechnol. 168(6), 1718-1727 (2012).

56 Cuevas-Cordoba B, Santiago-Garcia J. Saliva: a fluid of study for OMICS. Omics 18(2), 87-97 (2014).

57 Mikkonen JJ, Singh SP, Herrala M, Lappalainen R, Myllymaa S, Kullaa AM. Salivary metabolomics in the diagnosis of oral cancer and periodontal diseases. J. Periodontal Res. doi:10.1111/jre.12327 (2015) (Epub ahead of print).

58 Schafer CA, Schafer JJ, Yakob M, Lima P, Camargo P, Wong DT. Saliva diagnostics: utilizing oral fluids to determine health status. Monogr. Oral Sci. 24, 88-98 (2014).

59 Shankar AA, Alex S, Routray S. Incorporation of salivary metabolomics in oral cancer diagnostics. Oral Oncol. 50 (10), e53-e54 (2014).

60 Sugimoto M, Wong DT, Hirayama A, Soga T, Tomita M. Capillary electrophoresis mass spectrometry-based saliva metabolomics identified oral, breast and pancreatic cancerspecific profiles. Metabolomics 6(1), 78-95 (2010).

61 Barnes VM, Kennedy AD, Panagakos F et al. Global metabolomic analysis of human saliva and plasma from healthy and diabetic subjects, with and without periodontal disease. PLoS ONE 9(8), e105181 (2014).

62 de Laurentiis G, Paris D, Melck D et al. Separating smokingrelated diseases using NMR-based metabolomics of exhaled breath condensate. J. Proteome Res. 12(3), 1502-1511 (2013).

63 Mook-Kanamori DO, Selim MM, Takiddin AH et al. 1,5-Anhydroglucitol in saliva is a noninvasive marker of short-term glycemic control. J. Clin. Endocrinol. Metabol. 99(3), E479-E483 (2014).

64 Takayama T, Kuwabara T, Maeda T et al. Profiling of chiral and achiral carboxylic acid metabolomics: synthesis and evaluation of triazine-type chiral derivatization reagents for carboxylic acids by LC-ESI-MS/MS and the application to saliva of healthy volunteers and diabetic patients. Anal. Bioanal. Chem. 407(3), 1003-1014 (2015).

65 Kageyama G, Saegusa J, Irino Y et al. Metabolomics analysis of saliva from patients with primary Sjogren's syndrome. Clin. Exp. Immunol. 182(2), 149-153 (2015).

66 Tsuruoka M, Hara J, Hirayama A et al. Capillary electrophoresis-mass spectrometry-based metabolome analysis of serum and saliva from neurodegenerative dementia patients. Electrophoresis 34(19), 2865-2872 (2013)

67 Yan SK, Wei BJ, Lin ZY, Yang Y, Zhou ZT, Zhang WD. A metabonomic approach to the diagnosis of oral squamous cell carcinoma, oral lichen planus and oral leukoplakia. Oral Oncol. 44(5), 477-483 (2008).

68 Wei J, Xie G, Zhou Z et al. Salivary metabolite signatures of oral cancer and leukoplakia. Int. J. Cancer 129(9), 2207-2217 (2011).

69 Wang Q, Gao P, Wang X, Duan Y. The early diagnosis and monitoring of squamous cell carcinoma via saliva metabolomics. Sci. Rep. 4, 6802 (2014). 
70 Bos LD, Van Walree IC, Kolk AH, Janssen HG, Sterk PJ, Schultz MJ. Alterations in exhaled breath metabolitemixtures in two rat models of lipopolysaccharide-induced lung injury. J. Appl. Physiol. 115(10), 1487-1495 (2013).

71 de Laurentiis G, Paris D, Melck D et al. Metabonomic analysis of exhaled breath condensate in adults by nuclear magnetic resonance spectroscopy. Eur. Respir. J. 32(5), 1175-1183 (2008).

72 Basanta M, Jarvis RM, Xu Y et al. Non-invasive metabolomic analysis of breath using differential mobility spectrometry in patients with chronic obstructive pulmonary disease and healthy smokers. Analyst 135(2), 315-320 (2010).

73 Fens N, Douma RA, Sterk PJ, Kamphuisen PW. Breathomics as a diagnostic tool for pulmonary embolism. J. Thromb. Haemost. 8(12), 2831-2833 (2010).

74 Sofia M, Maniscalco M, De Laurentiis G, Paris D, Melck D, Motta A. Exploring airway diseases by NMR-based metabonomics: a review of application to exhaled breath condensate. J. Biomed. Biotechnol. 2011403260 (2011).

75 Guallar-Hoyas C, Turner MA, Blackburn GJ, Wilson ID, Thomas CL. A workflow for the metabolomic/metabonomic investigation of exhaled breath using thermal desorption GC-MS. Bioanalysis 4(18), 2227-2237 (2012).

76 Bos LD, Weda H, Wang Y et al. Exhaled breath metabolomics as a noninvasive diagnostic tool for acute respiratory distress syndrome. Eur. Respir. J. 44(1), 188-197 (2014).

77 Van Der Schee MP, Paff T, Brinkman P, Van Aalderen WM, Haarman EG, Sterk PJ. Breathomics in lung disease. Chest 147(1), 224-231 (2015).

78 Motta A, Paris D, Melck D et al. Nuclear magnetic resonance-based metabolomics of exhaled breath condensate: methodological aspects. Eur. Respir. J. 39 (2), 498-500 (2012).

79 Krilaviciute A, Heiss JA, Leja M, Kupcinskas J, Haick H, Brenner H. Detection of cancer through exhaled breath: a systematic review. Oncotarget 6(36), 38643-38657 (2015).

-. Provides comprehensive information on cancer detection through exhaled breath analysis.

80 Carraro S, Giordano G, Reniero F et al. Asthma severity in childhood and metabolomic profiling of breath condensate. Allergy 68(1), 110-117 (2013).

81 Gahleitner F, Guallar-Hoyas C, Beardsmore CS, Pandya HC, Thomas CP. Metabolomics pilot study to identify volatile organic compound markers of childhood asthma in exhaled breath. Bioanalysis 5(18), 2239-2247 (2013).

82 Ibrahim B, Marsden P, Smith JA, Custovic A, Nilsson M, Fowler SJ. Breath metabolomic profiling by nuclear magnetic resonance spectroscopy in asthma. Allergy 68(8), 1050-1056 (2013).

83 Motta A, Paris D, D'Amato M et al. NMR metabolomic analysis of exhaled breath condensate of asthmatic patients at two different temperatures. J. Proteome Res. 13(12), 6107-6120 (2014).

84 Carraro S, Rezzi S, Reniero F et al. Metabolomics applied to exhaled breath condensate in childhood asthma. Am. J. Respir. Crit. Care Med. 175(10), 986-990 (2007).
85 Mangler M, Freitag C, Lanowska M, Staeck O, Schneider A, Speiser D. Volatile organic compounds (VOCs) in exhaled breath of patients with breast cancer in a clinical setting. Ginekol. Pol. 83(10), 730-736 (2012).

86 Li J, Peng Y, Liu Y et al. Investigation of potential breath biomarkers for the early diagnosis of breast cancer using gas chromatography-mass spectrometry. Clin. Chim. Acta 436 59-67 (2014).

87 Montuschi P, Paris D, Melck D et al. NMR spectroscopy metabolomic profiling of exhaled breath condensate in patients with stable and unstable cystic fibrosis. Thorax 67(3), 222-228 (2012).

88 Monge ME, Perez JJ, Dwivedi P et al. Ion mobility and liquid chromatography/mass spectrometry strategies for exhaled breath condensate glucose quantitation in cystic fibrosis studies. Rapid Comm. Mass Spec. 27(20), 2263-2271 (2013).

89 Qin T, Liu H, Song Q et al. The screening of volatile markers for hepatocellular carcinoma. Cancer Epidemiol. Biomark. Prev. 19(9), 2247-2253 (2010).

90 Kalluri U, Naiker M, Myers MA. Cell culture metabolomics in the diagnosis of lung cancer-the influence of cell culture conditions. J. Breath Res. 8(2), 027109 (2014).

91 Handa H, Usuba A, Maddula S, Baumbach JI, Mineshita M, Miyazawa T. Exhaled breath analysis for lung cancer detection using ion mobility spectrometry. PLoS ONE 9(12), e114555 (2014).

92 Song G, Qin T, Liu H et al. Quantitative breath analysis of volatile organic compounds of lung cancer patients. Lung Cancer 67(2), 227-231 (2010).

93 Wang Y, Hu Y, Wang D et al. The analysis of volatile organic compounds biomarkers for lung cancer in exhaled breath, tissues and cell lines. Cancer Biomark. 11(4), 129-137 (2012).

94 Zou Y, Zhang X, Chen X, Hu Y, Ying K, Wang P. Optimization of volatile markers of lung cancer to exclude interferences of non-malignant disease. Cancer Biomark. 14(5), 371-379 (2014).

95 Lamote K, Nackaerts K, Van Meerbeeck JP. Strengths, weaknesses, and opportunities of diagnostic breathomics in pleural mesothelioma-a hypothesis. Cancer Epidemiol. Biomark. Prev. 23(6), 898-908 (2014).

96 Montuschi P, Paris D, Montella S et al. Nuclear magnetic resonance-based metabolomics discriminates primary ciliary dyskinesia from cystic fibrosis. Am. J. Respir. Crit. Care Med. 190 (2), 229-233 (2014).

97 Fowler SJ, Basanta-Sanchez M, Xu Y, Goodacre R, Dark PM. Surveillance for lower airway pathogens in mechanically ventilated patients by metabolomic analysis of exhaled breath: a case-control study. Thorax 70 (4), 320-325 (2015).

98 Bezabeh T, Somorjai RL, Smith IC. MR metabolomics of fecal extracts: applications in the study of bowel diseases. Magn. Res. Chem. 47(Suppl. 1), S54-S61 (2009).

99 Gao X, Pujos-Guillot E, Martin JF et al. Metabolite analysis of human fecal water by gas chromatography/mass spectrometry with ethyl chloroformate derivatization. Anal. Biochem. 393(2), 163-175 (2009). 
$100 \mathrm{Xu}$ W, Chen D, Wang N et al. Development of highperformance chemical isotope labeling LC-MS for profiling the human fecal metabolome. Anal. Chem. 87(2), 829-836 (2015).

101 Ahmed I, Greenwood R, Costello Bde L, Ratcliffe NM, Probert CS. An investigation of fecal volatile organic metabolites in irritable bowel syndrome. PLoS ONE 8(3), e58204 (2013).

102 Le Gall G, Noor SO, Ridgway K et al. Metabolomics of fecal extracts detects altered metabolic activity of gut microbiota in ulcerative colitis and irritable bowel syndrome. J. Proteome Res. 10(9), 4208-4218 (2011).

103 Monleon D, Morales JM, Barrasa A, Lopez JA, Vazquez C, Celda B. Metabolite profiling of fecal water extracts from human colorectal cancer. NMR Biomed. 22(3), 342-348 (2009).

104 Goedert JJ, Sampson JN, Moore SC et al. Fecal metabolomics: assay performance and association with colorectal cancer. Carcinogenesis 35 (9), 2089-2096 (2014).

105 Phua LC, Chue XP, Koh PK, Cheah PY, Ho HK, Chan EC. Non-invasive fecal metabonomic detection of colorectal cancer. Cancer Biol. Ther. 15(4), 389-397 (2014).

106 Walker A, Lucio M, Pfitzner B et al. Importance of sulfurcontaining metabolites in discriminating fecal extracts between normal and Type-2 diabetic mice. J. Proteome Res. 13(10), 4220-4231 (2014).

107 Inagaki S, Noda T, Min JZ, Toyo'oka T. Metabolic profiling of rat hair and screening biomarkers using ultra performance liquid chromatography with electrospray ionization time-offlight mass spectrometry. J. Chromatogr. A 1176(1-2), 94-99 (2007).

108 Gonska T, Ip W, Turner D et al. Sweat gland bioelectrics differ in cystic fibrosis: a new concept for potential diagnosis and assessment of CFTR function in cystic fibrosis. Thorax 64(11), 932-938 (2009).

109 Calderon-Santiago M, Priego-Capote F, Turck N et al. Human sweat metabolomics for lung cancer screening. Anal. Bioanal. Chem. 407(18), 5381-5392 (2015).

110 Chalasani N, Younossi Z, Lavine JE et al. The diagnosis and management of non-alcoholic fatty liver disease: practice Guideline by the American Association for the Study of Liver Diseases, American College of Gastroenterology, and the American Gastroenterological Association. Hepatology 55(6), 2005-2023 (2012).

111 Wruck W, Kashofer K, Rehman S et al. Multi-omic profiles of human non-alcoholic fatty liver disease tissue highlight heterogenic phenotypes. Sci. Data 2, 150068 (2015).

112 Chen L, Zhou L, Chan EC, Neo J, Beuerman RW. Characterization of the human tear metabolome by LC-MS/ MS. J. Proteome Res. 10 (10), 4876-4882 (2011).

113 Pieragostino D, D’Alessandro M, Di Ioia M, Di Ilio C, Sacchetta P, Del Boccio P. Unraveling the molecular repertoire of tears as a source of biomarkers: beyond ocular diseases. Proteomics Clin. Appl. 9(1-2), 169-186 (2015). 